\title{
Diagnostic and prognostic role of proton single-voxel spectroscopy (SVS) in non- lesional epilepsy pediatric patients: prospective controlled study
}

\author{
Mohamed H. Faheem ${ }^{1 *}$ (D, Ahmed S. Dabour ${ }^{2}$ and Omima M. Abdelhaie ${ }^{3}$
}

\begin{abstract}
Background: We aimed to verify the diagnostic and prognostic role of proton single-voxel spectroscopy (SVS) in children with non-lesional epilepsy and its add-on value to conventional MR. The prospective controlled study carried out on the epileptic patients who were regularly following in the pediatric neurology clinic in our university hospital, over the period from July 2017 to July 2018. It compared SVS findings (NAA/Cr, NAA/Cho, and NAA/ Cho+ $\mathrm{Cr}$ ratios) between the case (50 patients) and control group (20 children), between the cases with different seizures semiology and between the patients with intractable and non-intractable epilepsy.

Results: NAA/Cr ratio showed a significant difference between the patients with intractable and non-intractable epilepsy in the basal ganglia ( $P$ value 0.005 ) and white matter $(P$ value 0.043 ) with cutoff values of 1.5 and 1.9 respectively. A significant difference of NAA/Cho ratio was found between generalized seizures cases and other seizures semiology in basal ganglia ( $P$ value 0.012$)$ and cortex $(P$ value $<.001)$. There was no significant difference between the patient and control groups or between generalized seizures cases and the control group.

Conclusion: Proton SVS has limited diagnostic value in non-lesional epilepsy pediatric patients, in differentiation between generalized seizures and other seizure types, but, it has a good prognostic role in predicting patients who will develop intractable epilepsy.
\end{abstract}

Keywords: MR spectroscopy, Single-voxel spectroscopy, Epilepsy, Non-lesional epilepsy

\section{Background}

Epileptic seizures affect 1-2\% of the population and $4 \%$ of children. Childhood epilepsy has a prevalence of approximately $0.5-0.8 \%$ and comprises a heterogeneous group of disorders, including a variety of epilepsy syndromes that range in severity from benign to progressive and catastrophic [1].

According to ILAE (International League Against Epilepsy) "2014". Epilepsy is defined as a disease of the

\footnotetext{
* Correspondence: Dr.hosny.rad@gmail.com;

Mohamed.hosny@fmed.bu.edu.eg

${ }^{1}$ Radiology Department, Benha Faculty of Medicine, Benha University,

Kalyoubia, Egypt

Full list of author information is available at the end of the article
}

brain characterized by any of the following conditions: (a) at least two unprovoked (or reflex) seizures occurring $>24 \mathrm{~h}$ apart. (b) One unprovoked (or reflex) seizure and a probability of further seizures similar to the general recurrence risk (at least 60\%) after two unprovoked seizures, occurring over the next 10 years. (c) Diagnosis of an epilepsy syndrome [2].

Magnetic resonance imaging (MRI) plays a key role in the evaluation of pediatric patients with seizures and is considered important for the detection of epileptogenic lesions. However, magnetic resonance spectroscopy (MRS) is not as widely used for the evaluation of children with seizures and is not included in the ILAE

\section{Springer Open}

() The Author(s). 2020 Open Access This article is licensed under a Creative Commons Attribution 4.0 International License, which permits use, sharing, adaptation, distribution and reproduction in any medium or format, as long as you give appropriate credit to the original author(s) and the source, provide a link to the Creative Commons licence, and indicate if changes were made. The images or other third party material in this article are included in the article's Creative Commons licence, unless indicated otherwise in a credit line to the material. If material is not included in the article's Creative Commons licence and your intended use is not permitted by statutory regulation or exceeds the permitted use, you will need to obtain permission directly from the copyright holder. To view a copy of this licence, visit http://creativecommons.org/licenses/by/4.0/. 
recommendations for the management of infantile seizures again in 2015 [3].

Many authors studied the role of MRS in epilepsy, with temporal lobe epilepsy (TLE) had a great part of these works [4-8]. One study studied MRS in nonlesional insular cortex epilepsy. They used both singlevoxel spectroscopy (SVS) and multi-voxel spectroscopy techniques [9]. However, to our knowledge, no studies were dedicated to study SVS in non-lesional epilepsy in pediatric age group (after advanced research using PMC, pub Med, Google scholar and research gate data base up to November 2019).

Some studies used clinical parameters like type of seizures, cause of epilepsy, family history of epilepsy, semiology of seizures as a predictive value of intractable seizures [10]. Others postulated genetic factors. Unfortunately, none of them was approved as a definite predictor of treatment failure [11].

The goal of this prospective study was to determine whether SVS had additional value in children with nonlesional epilepsy. We correlated our results with the clinical findings and patients' response to therapy.

\section{Methods}

\section{Patients}

Our study was a prospective controlled study. It had been conducted on pediatric epileptic patients who were regularly following in the pediatric neurology clinic, in our university hospital. It was carried out over the period from July 2017 to July 2018 . The study sample included two groups; the case group (50 epileptic patients) and the control group (20 neurologically free children).

The inclusion criteria included: Children from 1 to 18 years old from both genders who were diagnosed with epilepsy-according to ILAE criteria [2] - and showed unremarkable conventional brain MRI non-lesional epilepsy).

The exclusion criteria included; the presence of contraindications to MRI (such as pacemakers and cochlear implants) and epileptic children who had lesions in conventional brain MRI.

\section{Methodology}

Written consents for the study were obtained from the parents after a full explanation of the study procedures. The consents were obtained prior to the children's enrollment in the study. The study protocol was approved by the Ethical Committees of our faculty.

The control group was chosen from other children presented to the pediatric outpatient clinic in our hospital after making sure that they were neurologically free. Their parents's acceptance was requested and informed consent for the participation was taken.

All the subjects in the cases group underwent the following: Full history taking with special emphasis on perinatal history, developmental history, history of consanguinity, family history of epilepsy or febrile convulsion,

\section{ILAE 2017 Classification of Seizure Types Expanded Version ${ }^{1}$}

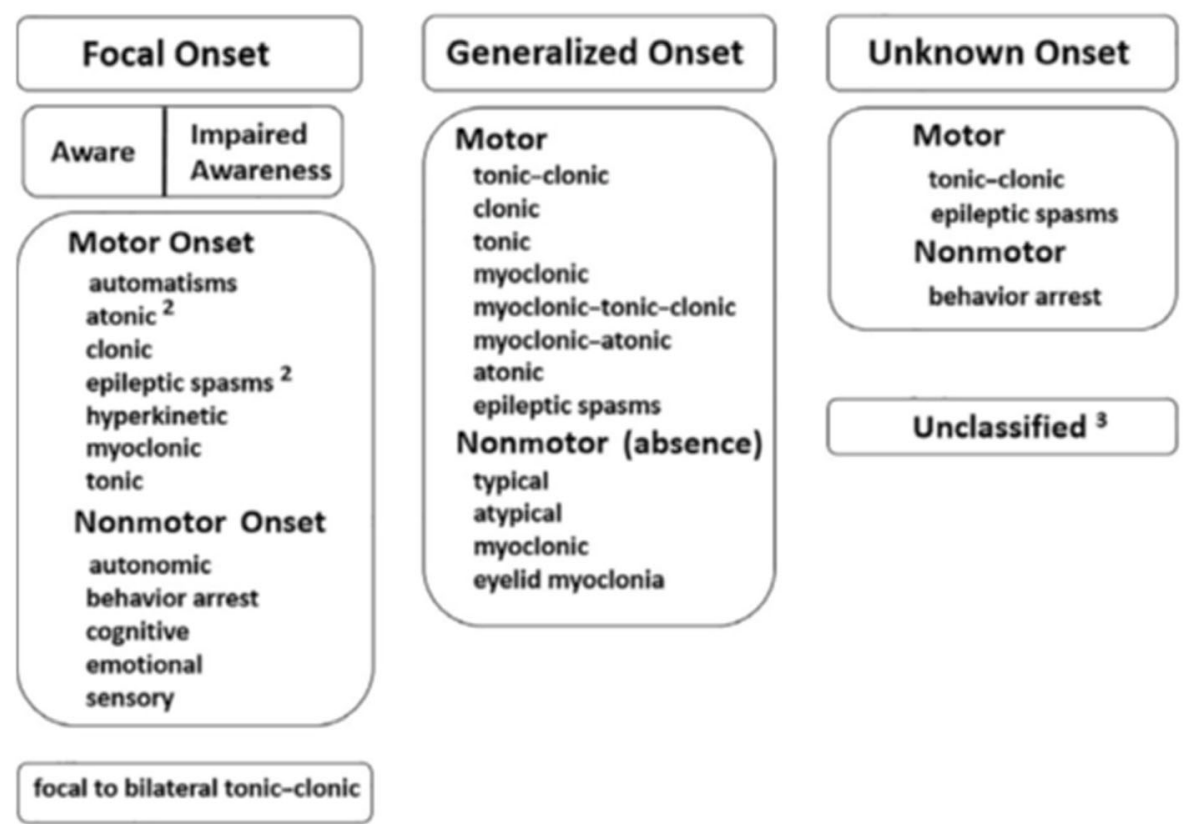

Fig. 1 The expanded ILAE 2017 operational classification of seizure types [12] 
semiology of the seizures, the age of onset, type of antiepileptic drugs, response to treatment, seizure frequency, compliance to treatment.

Classification of seizure types according to the revised operational classification done by ILAE in 2017 (figure 1) [12].

According to the response to medical treatment, the cases group was divided into two sub-groups: non-intractable and intractable cases. The intractable (drugresistant) epilepsy was defined as "failure of adequate trials of two tolerated, appropriately chosen and used antiepileptic drug schedules (whether as monotherapies or in combination) to achieve sustained seizure freedom [13].

Physical examination (general and local): including a full neurological examination.

All the cases in each group were subjected to the following Conventional MRI: MRI imaging was held using $1.5 \mathrm{~T}$ (Siemens, MAGNETOM Aera) machine, in MRI unit, radiology department, in our university hospital. The MR exam was done at least 2 weeks post last seizure attack to avoid the effect of postictal metabolic changes. The conventional MR study included the following sequences; axial and sagittal T1WIs SE (TE $8.9 \mathrm{~ms}$, TR $450 \mathrm{~ms}$ ), axial T2WIs TSE (TE $113 \mathrm{~ms}$, TR $4490 \mathrm{~ms}$ ), axial and coronal FLAIR (TE $107 \mathrm{~ms}$, TR $9000 \mathrm{~ms}$, TI 2500 and flip angle $150^{\circ}$ ), and coronal $\mathrm{T} 1$ inversion recovery images (TE 66 ms, TR $7000 \mathrm{~ms}$, TI 350 and flip angle $160^{\circ}$ ).

SVS: voxels were placed at the left centrum semiovale, the left basal ganglia, and the midline parieto-occipital regions as representers of the white matter, deep grey matter, and cortical grey matter respectively. The examination was done using short echo time TE $(30 \mathrm{~ms})$ and long echo time TE (135 ms) with voxel size $2-5 \mathrm{ml}$ (Fig. 2).

Both the MRI and SVS were done in the same setting. Children $<7$ years old and uncooperative ones were sedated either using chloral hydrate in a dose of $0.1 \mathrm{~g} / \mathrm{kg}$ or using midazolam injection in a dose of $0.1 \mathrm{mg} / \mathrm{kg}$ together with propofol injection in a dose of $1 \mathrm{mg} / \mathrm{kg}$ followed by $0.5 \mathrm{mg} / \mathrm{kg}$ if necessary.

The integral values of various metabolite peaks were evaluated in every voxel. Three important metabolites resonance were identified: NAA at $2.02 \mathrm{ppm}, \mathrm{Cr}$ at 3.02 ppm, and Cho at $3.22 \mathrm{ppm}$.

The NAA/Cho ratio, the NAA/Cr ratio and the NAA/ $\mathrm{Cr}+\mathrm{Cho}$ ratios were calculated in the three different sites (the white matter, basal ganglia (Fig. 3), and cortical grey matter).

\section{Statistical methods}

- Data management and statistical analysis were done using SPSS vs.25. (IBM, Armonk, New York, United States).
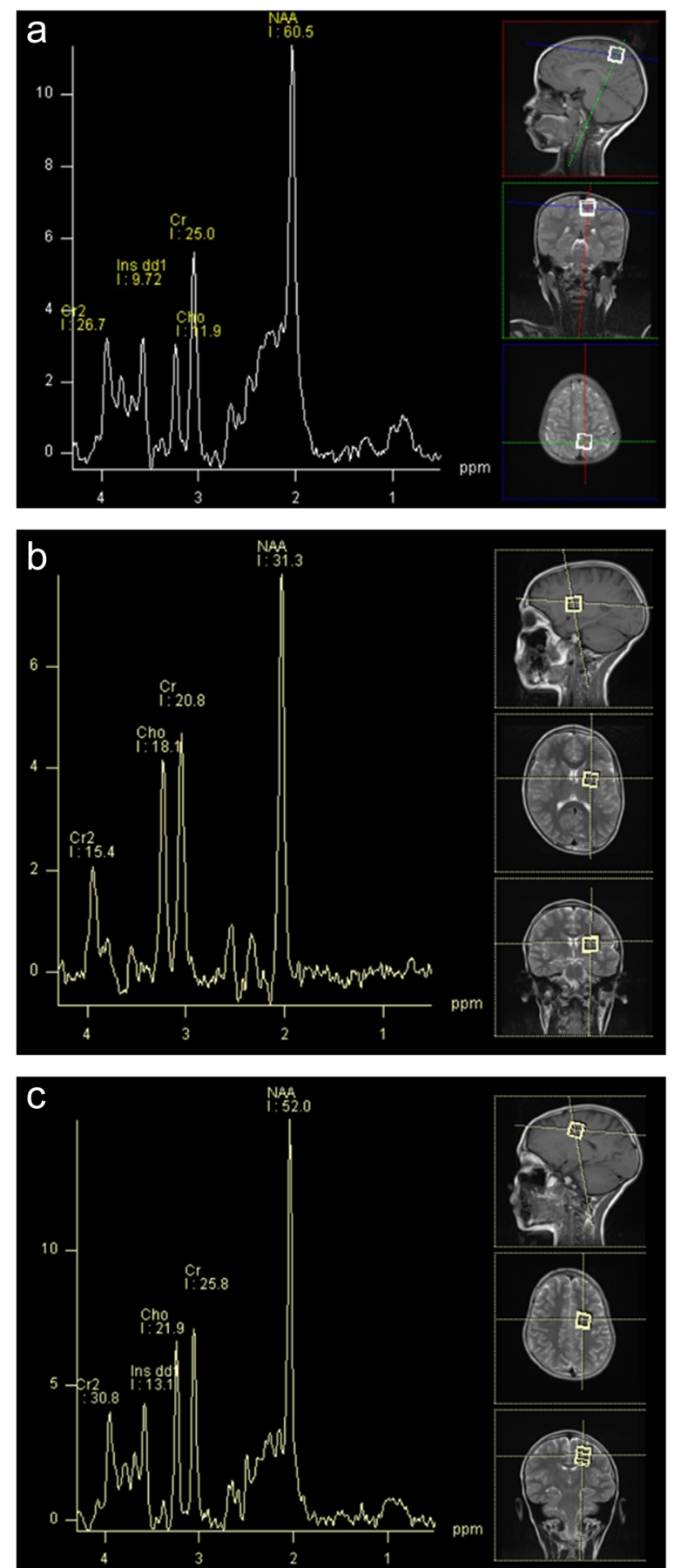

Fig. 2 a Showing single-voxel placement in the midline parietooccipital region. b Showing single-voxel placement in the left basal ganglia region (centered on lentiform nucleus). c Showing singlevoxel placement in the left centrum semiovale

- Numerical data was summarized as means and standard deviations. Categorical data was summarized as numbers and percentages.

- Comparisons between two groups were done using Mann Whitney U test for numerical data. 
Categorical data was compared using chi-square test or Fisher's exact test if appropriate.

- Correlation analysis between different metabolites was done using Pearson's correlation. " $r$ " is the correlation coefficient. It ranges from -1 to $+1 .-1$ indicates a strong negative correlation, +1 indicates a strong positive correlation while 0 indicates no correlation.

- All P values were two-sided. $P$ values less than 0.05 were considered significant.

\section{Results}

Seventy persons were included in the current study (50 cases and 20 controls). Their epidemiological characters were illustrated in Table 1 . There was no significant statistical difference between case and control groups regarding their age, sex, and family history of epilepsy.

According to the type of seizures, 44 (88\%) cases presented with generalized seizures, while each of focal seizures, focal seizures with impaired awareness, and myoclonic seizures were presented by $2(4 \%)$ cases. According to seizures control, $8(16 \%)$ of cases presented with intractable epilepsy while other cases (42 cases "84\%") were non-intractable.

No abnormal metabolite peaks were found in the short TE spectra of all cases group.

We did not find significant statistical differences between cases and control groups regarding the calculated $\mathrm{NAA} / \mathrm{Cho}, \mathrm{NAA} / \mathrm{Cr}$, and $\mathrm{NAA} / \mathrm{Cr}+\mathrm{Cho}$ ratios in the basal ganglia, white matter, and cortical grey matter $(P$ values for NAA/Cho ratio were $0.268,0.629$, and 0.467 , $P$ values for $\mathrm{NAA} / \mathrm{Cr}$ ratio were $0.281,0.131$, and 0799 and $P$ values for NAA/ Cho+Cr ratio were $0.852,0.797$, and 0.272 respectively).

There were no significant statistical differences between the cases with intractable seizures and those with non-intractable seizures regarding the calculated NAA/ Cho ratio in basal ganglia, white matter, and cortical grey matter $(P$ values $0.687,0.254$, and 0.482 respectively). However, the calculated $\mathrm{NAA} / \mathrm{Cr}$ ratio showed statistically significant differences between the two groups in the basal ganglia and white matter. There was borderline significance found in cortical grey matter (Table 2).

Our results also showed a borderline significant statistical difference between cases with intractable seizures and those with non-intractable seizures regarding the calculated NAA/Cr+Cho ratio only in basal ganglia $(P$ value 0.073 ), with no statistical difference noted in white matter and cortical grey matter $(P$ values 0.345 and 0.297 respectively).

The present work revealed statistically significant differences between patients with generalized seizures and those with other seizure semiology regarding the calculated NAA/Cho ratio in basal ganglia $(P$ value $=0.012)$ and cortical grey matter $(P$ value $<0.001)$, but no statistically significant differences noted in the white matter $(P$ value $=0.812$ ). In contrast, there was a statistically significant difference between the two groups regarding the calculated NAA/Cr ratio in white matter $(P$ value $=$ 0.03 ), yet no significant difference could be noted in the cortical grey matter and the basal ganglia $(P$ values $=$ 0.122 and 0.388 respectively). Also, we found statistically significant differences between the two groups regarding the calculated NAA/Cr+Cho ratio in cortical grey matter

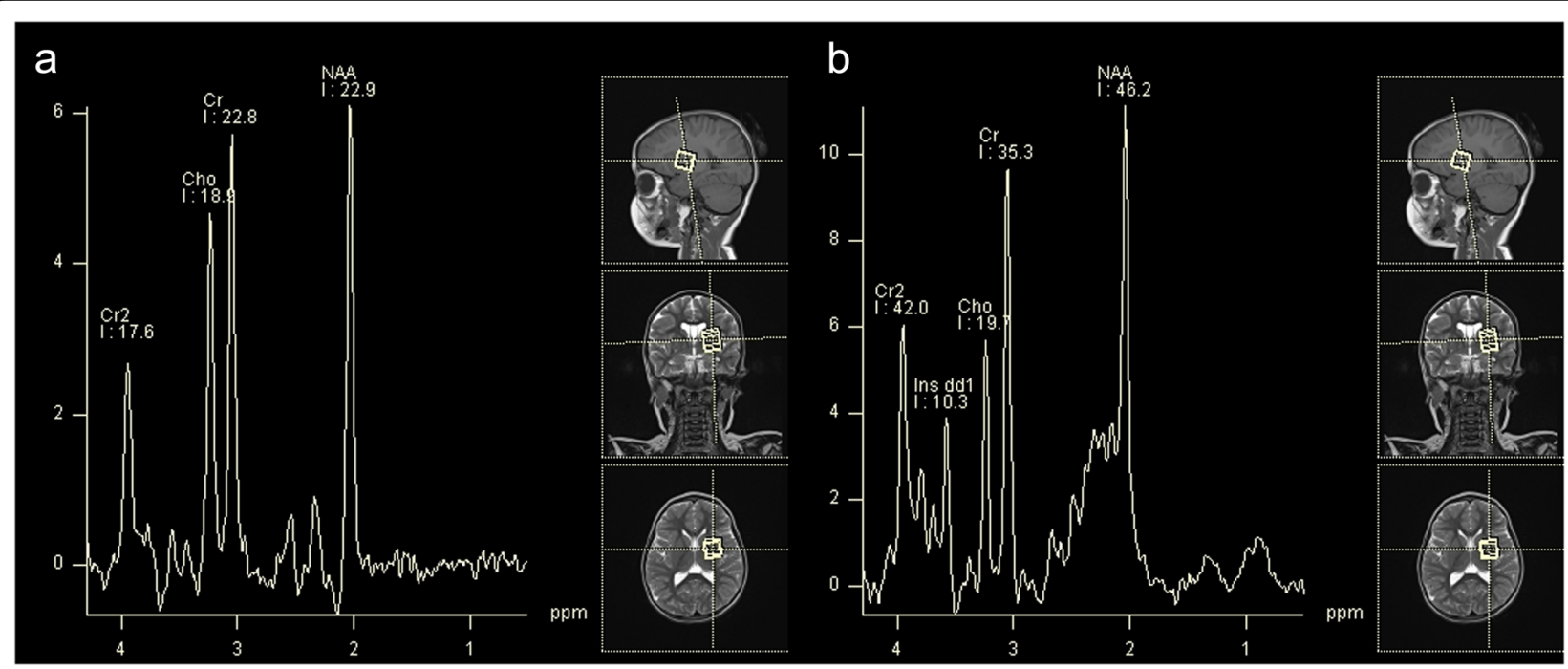

Fig. 3 Long (a) and short (b) TE SVS with voxel placed in the left lentiform nucleus, in a 5-years old female presented by intractable generalized seizures showing no abnormal peaks in the short TE spectrum. The long TE spectrum showed NAA/Cr=1, NAA/Cho=1.2, and NAA/Cho+Cr=0.54 
Table 1 General characteristics of case and control groups

\begin{tabular}{lllll}
\hline & & Cases $(n=50)$ & Controls $(n=20)$ & $P$ value \\
\hline Age (years) & Mean \pm SD & $5 \pm 3$ & $6 \pm 4$ & $11(55.0)$ \\
Gender & Males $n(\%)$ & $30(60.0)$ & $9(45.0)$ & 0.701 \\
Family history of epilepsy & Females $n(\%)$ & $20(40.0)$ & $0(0.0)$ & 1.0 \\
\hline
\end{tabular}

$(P$ value $=0.016)$ with no significant difference noted in the white matter and basal ganglia $(P$ values $=0.108$ and 0.125 respectively).

The calculated NAA/Cr, NAA/Cho, and NAA/Cho+ $\mathrm{Cr}$ ratios within the basal ganglia, white matter, and cortical grey matter showed no significant difference between the patients with generalized convulsions and the control group (Table 3).

ROC curve was calculated for $\mathrm{NAA} / \mathrm{Cr}$ ratio in basal ganglia and gave cutoff value $\leq 1.5$ for epileptic patients who could respond to medical treatment from those with intractable seizures, at maximum combined sensitivity and specificity of $100 \%$ and $62 \%$ respectively and area under the curve of $0.81 ;(P<0.05)$ (Fig. 4).

ROC curve was also done for the NAA/Cr ratio in white matter and found cutoff value $\leq 1.9$ could differentiate epileptic children who will respond to treatment from those with intractable seizures, with best-combined sensitivity and specificity of $100 \%$ and $65 \%$ respectively. AUC $=0.758 ;(P<0.05)$ (Fig. 5).

\section{Discussion}

In the current study, the case group included 50 epileptic patients, 30 males (60\%), and 20 females (40\%) with mean age of $5 \pm 3$ years. The control group included 20 patients, 11 males (55\%), and 9 females (45\%) with mean age of $6 \pm 4$ years. For most recent studies that reported gender, like the study conducted by Hawley et al. in 2015, gender-specific incidence in children was higher in males, although seldom statistically significant [14].

The case group included 8 patients (16\%) with intractable epilepsy and 42 patients (84\%) who were controlled with medication. This nearly agreed with the Dutch study by Arts et al. conducted as a 5-year follow-up study of epilepsy in childhood who found that $6-4 \%$ of children developed intractable epilepsy [15].
In the current study, the ILAE 2017 classification of seizure types was used. The most frequent type of seizures was generalized tonic-clonic seizures $88 \%$ followed by focal seizures with awareness which represented $4 \%$, focal seizures with impaired awareness $4 \%$, and myoclonic seizures which also represented $4 \%$. This went with Farghaly et al., who conducted a study to determine the prevalence and clinical pattern of epilepsy among children and adolescents in upper Egypt. This study demonstrated generalized seizures as the most frequent type in epileptic children and adolescents [16].

In the present study, we used SVS technique, as it offered a good signal to noise and homogenous peaks within relatively short scan time [17]. We did not add a multivoxel technique in this study to avoid unnecessary prolongation of the exam time.

The quality of the signal received from metabolites in proton MRS directly depends on the physical parameters of the impulse sequence used, namely on Echo time (TE). In this study, we used short (30 ms) and long (135 $\mathrm{ms}$ ) TE. As short TE allowed us to detect more metabolites especially myo-inositol and glutamates, As the change of their levels found to be related to poor outcome in some cases [18]. We also used long TE because the attenuation of the signal from the metabolites with shorter time of relaxation (TR), improves the signalnoise ratio of the main three metabolites (NAA, Cho, and $\mathrm{Cr}$ ), and allowed better calculation of the ratios [19].

In their review article, Caruso et al. suggested one of two SVS protocols for the examination of the patients with non-lesional epilepsy. One protocol was to survey white matter, deep and cortical grey matter by using 3 voxels-using short TE of $35 \mathrm{~ms}$-over the centrum semiovale, lentiform nuclei, and midline occipito-parietal cortex. Another protocol was using one voxel over the basal ganglia in order to save time [18]. In the present study, we went with Caruso's first protocol, we placed

Table 2 NAA/Cr in basal ganglia, white matter, and grey matter according to seizures control

\begin{tabular}{llll}
\hline & Intractable $(n=8)$ & Non-intractable $(n=42)$ & Mean \pm SD \\
& Mean \pm SD & $1.67 \pm 0.31$ & $0.005^{*}$ \\
\hline In basal ganglion & $1.39 \pm 0.12$ & $1.89 \pm 0.34$ & $0.043^{*}$ \\
In white matter & $1.69 \pm 0.21$ & $1.87 \pm 0.29$ & $0.06^{* *}$ \\
In grey matter & $1.67 \pm 0.18$ &
\end{tabular}

*Significant, **borderline significant 
Table $3 \mathrm{NAA} / \mathrm{Cr}$, NAA/Cho, and NAA/Cho+Cr in basal ganglia, white matter, and cortical grey matter between the patients with generalized seizures and the control group

\begin{tabular}{|c|c|c|c|c|c|}
\hline & \multicolumn{2}{|c|}{ Generalized seizures } & \multicolumn{2}{|c|}{ Controls } & \multirow[b]{2}{*}{$P$ value } \\
\hline & Mean & $\pm \mathrm{SD}$ & Mean & $\pm \mathrm{SD}$ & \\
\hline \multicolumn{6}{|l|}{ NAA/Cho } \\
\hline Basal ganglion & 2.33 & 0.56 & 2.24 & 0.49 & 0.482 \\
\hline White matter & 1.91 & 0.37 & 1.86 & 0.25 & 0.586 \\
\hline Grey matter & 2.81 & 0.79 & 2.79 & 1.23 & 0.901 \\
\hline \multicolumn{6}{|l|}{$\mathrm{NAA} / \mathrm{Cr}$} \\
\hline Basal ganglion & 1.6 & 0.29 & 1.69 & 0.23 & 0.181 \\
\hline White matter & 1.9 & 0.31 & 1.69 & 0.39 & 0.069 \\
\hline Grey matter & 1.8 & 0.25 & 1.85 & 0.55 & 0.934 \\
\hline \multicolumn{6}{|l|}{$N A A / C r+c h o$} \\
\hline Basal ganglion & 0.96 & 0.15 & 1.65 & 2 & 1 \\
\hline White matter & 0.975 & 0.19 & 1.246 & 0.835 & 0.942 \\
\hline Grey matter & 1.11 & 0.18 & 1.33 & 0.48 & 0.153 \\
\hline
\end{tabular}

one voxel in the left centrum semiovale, one in the left lentiform nucleus, and one in the midline parietooccipital region.

In the current study, the compared NAA/Cho, NAA/ $\mathrm{Cr}$, and NAA/Cr+Cho ratios between the cases and control groups (in the different sampled sites) showed no significant difference. These findings denied the value of these ratios in identifying the patients with non-lesional epilepsy. This went with Aitouche et al. who found that the measurement of absolute NAA and NAA/Cr ratio cannot differentiate between non-lesional insular epilepsy, extra insular epilepsy, and control groups [9].
On the other hand, the present results were in disagreement with Azab et al. who compared the NAA/Cr ratio between 40 TLE patients and 20 controls. They found a significant difference between the patients and control groups with a decreased level of the NAA/Cr ratio in the patient's group [8]. Also, Aun et al. conducted a study to evaluate the role of MRS in non-lesional TLE. They found that low $\mathrm{NAA} / \mathrm{Cho}+\mathrm{Cr}$ ratio allowed for lateralization of the epileptogenic focus in comparing both temporal lobes [7]. We did not go with Cendes et al. in their meta-analysis, as they found that proton MRS studies showed a focal reduction of NAA signal in patients with different forms of TLE, including those with normal magnetic resonance imaging (MRI), as well as extratemporal partial epilepsies [20]. This contradiction may be explained as this study was conducted on non-lesional epilepsy without identification of the epileptogenic focus, while other studies were conducted over epilepsy in which epileptogenic foci were detectable in conventional MRI, EEG, and/or fluoro-deoxyglucose-positron emission tomography (FDG-PET).

In comparing metabolite ratios between groups with intractable and non-intractable epilepsy, the NAA/Cr ratio showed a statistically significant difference in basal ganglia, white matter, and grey matter. We also found a borderline significant difference between $\mathrm{NAA} / \mathrm{Cr}+\mathrm{Cho}$ ratio in basal ganglia. Our results pointed to the value of $\mathrm{NAA} / \mathrm{Cr}$ and $\mathrm{NAA} / \mathrm{Cr}+\mathrm{Cho}$ ratios as biomarkers that can predict the patient's response to treatment. This agreed with the study done by Campos et al. who demonstrated a significant reduction of NAA/ $\mathrm{Cr}$ ratios in interactable TLE group compared with those who responded to the first antiepileptic drugs (AED) and to

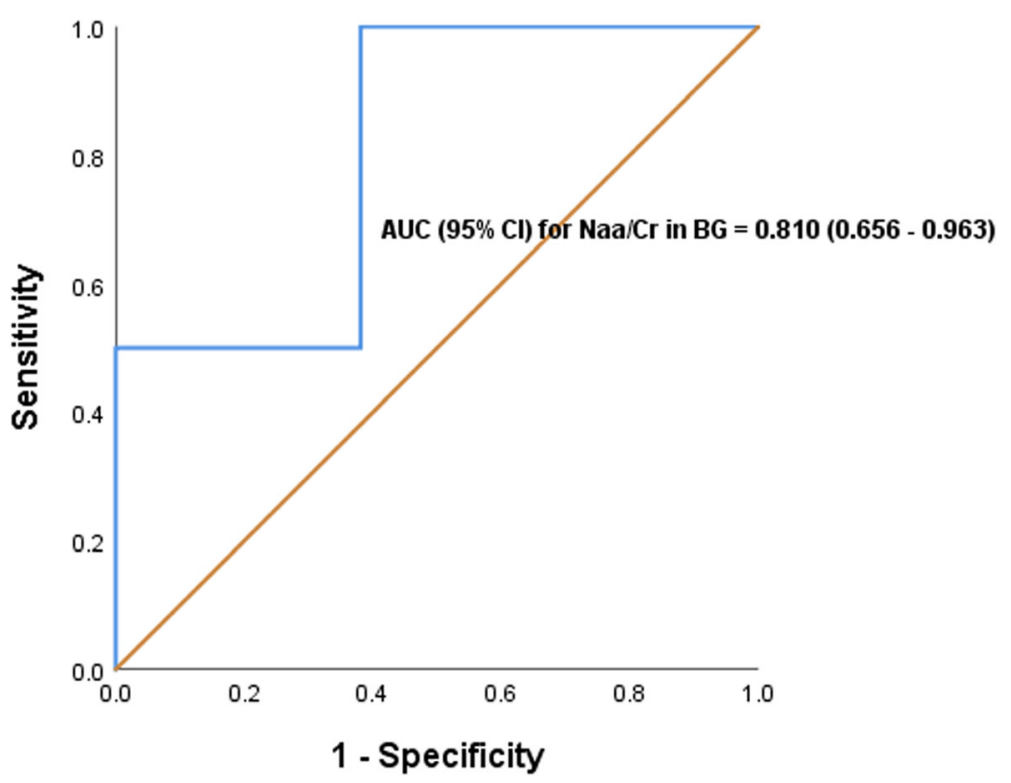

Fig. $4 \mathrm{ROC}$ analysis for NAA/Cr in BG for prediction of intractable seizures 


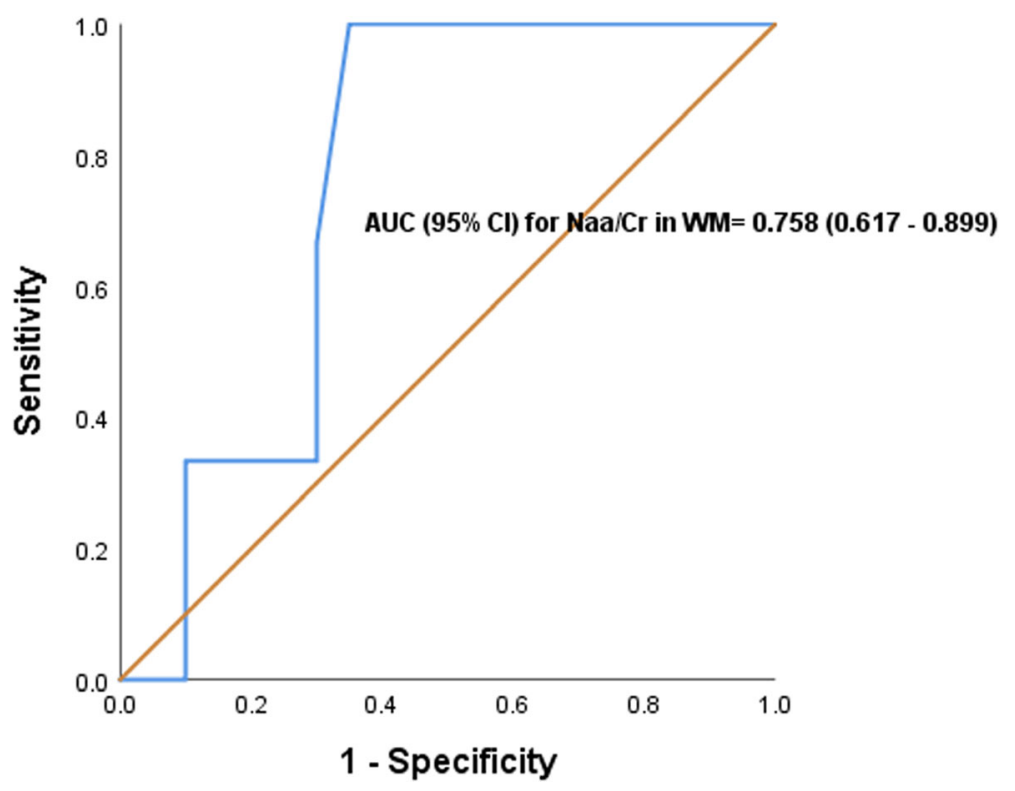

Fig. 5 ROC analysis for NAA/Cr in WM for prediction of intractable seizures

controls [21]. Also, this went with Briellmann et al. who found that NAA was significantly lower in refractory TLE patients compared with patients who responded to one anti-epileptic drugs [22].

This can be explained by the fact that NAA is considered a neuronal marker as it is found exclusively in neurons and their processes. In human brain spectra, NAA is reduced in the situations associated with neuronal loss or neuronal dysfunction. In patients with intractable seizures, the high frequency of breakthrough seizures, the more duration of the attacks, and the multiple antiepileptic drugs, all of these contribute to neuronal dysfunction [23].

In the present study, there were statistically significant differences between patients with generalized clonic seizures and those with other seizure semiology in NAA/ Cho ratio in basal ganglia and grey matter, $\mathrm{NAA} / \mathrm{Cr}$ ratio in white matter, and $\mathrm{NAA} / \mathrm{Cr}+\mathrm{Cho}$ ratio in the cortex. However, these ratios showed no significant difference between the patients with generalized seizures and the control group. This disagreed with Kasper et al. who used multi-voxel MRS to study the extent of local metabolic alterations in patients with idiopathic generalized epilepsy. They depended on the absolute values of the metabolites rather than ratios. They found a significant decrease $(P<$ 0.05 ) of tNAA in the cortical grey matter, thalamus, and basal ganglia, compared with the control group [24].

\section{Limitation}

- This study was done using magnetic field strength of 1.5 T. A higher-field strength of $3 \mathrm{~T}$ or more would yield a better spatial resolution and high SNR.
- Small sample size may reduce or bias the power of the results. Further studies with a larger sample size are necessary to extend these results.

\section{Conclusion}

SVS has limited diagnostic value in children with nonlesional epilepsy as it could differentiate between patients with generalized seizures and those with other seizure types, but it has a good prognostic tool in predicting patients who will develop intractable epilepsy.

\section{Abbreviations}

AED: Antiepileptic drugs; FLAIR: Fluid attenuation inversion recovery; ILAE: International League Against Epilepsy; MRI: Magnetic resonance imaging; MRS: Magnetic resonance spectroscopy; SV: Single-voxel spectroscopy; TE: Echo time; TI: Inversion time; TR: Repetition time; TLE: Temporal lobe epilepsy

\section{Acknowledgements}

We present sincere thanks to M. Bendary for his biostatistical support.

\section{Authors' contributions}

$\mathrm{AD}$ and $\mathrm{OA}$ carried up the clinical assessment of the epileptic patients and categorized the sample according to the seizures semiology and treatment response. MF supervised the MRS exams and interpreted their findings. All authors have read and approved the manuscript.

Funding

No funds were received for this study.

Availability of data and materials

The datasets used and/or analyzed during the current study are available from the corresponding author on reasonable request.

Competing interest

The authors declare that they have no competing interests in this section. 


\section{Ethics approval and consent to participate}

Written consents for the study were obtained from the parents after full explanation of the study procedures. The consents were obtained prior to the children's enrollment in the study. The study protocol was approved by the Ethical Committees of Benha faculty of medicine ( The reference number of the ethical committee of Benha Faculty of Medicine is not available till now. I contacted the responsible person of the committee and she said that they are taking the steps nowadays to have a reference number in the international research board 'IRB', but they have not finished yet)

\section{Consent for publication}

The parents of the cases were consented to publish the study data with the maintenance of full anonymity.

\section{Author details}

${ }^{1}$ Radiology Department, Benha Faculty of Medicine, Benha University, Kalyoubia, Egypt. ${ }^{2}$ Pediatric Neurology Unit, Pediatric Department, Benha Faculty of Medicine, Benha University, Kalyoubia, Egypt. ${ }^{3}$ Pediatric Department, Benha Faculty of Medicine, Benha University, Kalyoubia, Egypt.

Received: 23 March 2020 Accepted: 9 July 2020

Published online: 30 July 2020

\section{References}

1. Cross JH, Kluger G, Lagae $L$ (2013) Advancing the management of childhood epilepsies. Eur J Paediatr Neurol EJPN Off J Eur Paediatr Neurol Soc 17(4):334-347

2. Fisher RS, Cross JH, French JA, Higurashi N, Hirsch E, Jansen FE et al (2017) Operational classification of seizure types by the International League Against Epilepsy: Position Paper of the ILAE Commission for Classification and Terminology. Epilepsia 58(4):522-530

3. Rincon SP, Blitstein MBK, Caruso PA, González RG, Do RLT, Ratai E (2016) The use of magnetic resonance spectroscopy in the evaluation of pediatric patients with seizures. Pediatr Neurol 58:57-66

4. Aydin H, Oktay NA, Kizilgoz V, Altin E, Tatar I, Hekimoglu B (2012) Value of proton-MR-spectroscopy in the diagnosis of temporal lobe epilepsy; correlation of metabolite alterations with electroencephalography. Iran J Radiol 9(1):1-11

5. Ercan K, Gunbey HP, Bilir E, Zan E, Arslan H (2016) Comparative Lateralizing ability of multimodality MRI in temporal lobe epilepsy. Dis Markers.:1-9

6. Capizzano AA, Vermathen P, Laxer KD, Matson GB, Maudsley AA, Soher BJ et al (2002) Multisection proton MR spectroscopy for mesial temporal lobe epilepsy. AJNR Am J Neuroradiol 23(8):1359-1368

7. Aziz A, Aun K, Ahmed A, Mohamed A, Fotouh A, Saeed K et al (2016) Role of magnetic resonance spectroscopy (MRS) in nonlesional temporal lobe epilepsy. Egypt J Radiol Nucl Med 2016. 47(1):217-231

8. Azab SFA, Sherief LM, Saleh SH, Elshafeiy MM, Siam AG, Elsaeed WF et al (2015) Childhood temporal lobe epilepsy : correlation between electroencephalography and magnetic resonance spectroscopy : a case control study. Ital J Pediatr :41-32

9. Aitouche Y, Gibbs SA, Gilbert G, Boucher O, Bouthillier A, Nguyen DK (2017) Proton MR Spectroscopy in patients with nonlesional insular cortex epilepsy confirmed by invasive EEG recordings. J Neuroimaging Off J Am Soc Neuroimaging 27(5):517-523

10. Xue-Ping W, Hai-Jiao W, Li-Na Z, Xu D, Ling L (2019) Risk factors for drugresistant epilepsy: a systematic review and meta-analysis. Medicine (Baltimore) 98(30):e16402

11. Silva-Alves MS, Secolin R, Carvalho BS, Yasuda CL, Bilevicius E, Alvim MKM et al (2017) A prediction algorithm for drug response in patients with mesial temporal lobe epilepsy based on clinical and genetic information. Janigro D, editor. PLOS ONE 12(1):e0169214

12. Scheffer IE, Berkovic S, Capovilla G, Connolly MB, French J, Guilhoto L et al (2017) ILAE classification of the epilepsies: position paper of the ILAE commission for classification and terminology. Epilepsia 58(4):512-521

13. Kwan P, Arzimanoglou A, Berg AT, et al (2010) Definition of drug resistant epilepsy: consensus proposal by the ad hoc Task Force of the ILAE Commission on Therapeutic Strategies [published correction appears in Epilepsia. 2010 Sep;51(9):1922]. Epilepsia 51(6):1069-1077.

14. Hawley SR, Ablah E, Hesdorffer D, Pellock JM, Lindeman DP, Paschal AM et al (2015) Prevalence of pediatric epilepsy in low-income rural Midwestern counties. Epilepsy 53:190-196
15. Arts WFM, Brouwer OF, Peters ACB, Stroink H, Peeters EAJ, Schmitz PIM et al (2004) Course and prognosis of childhood epilepsy: 5-year follow-up of the Dutch study of epilepsy in childhood. Brain J Neurol 127(Pt 8):1774-1784

16. Farghaly WM, Abd Elhamed MA, Hassan EM, Soliman WT, Yhia MA, Hamdy NA (2018) Prevalence of childhood and adolescence epilepsy in Upper Egypt (desert areas). Egypt J Neurol Psychiatry Neurosurg 54(1):34

17. Cecil KM (2014) Protom Magnetic resonance spectroscopy: Technique for the neuroradiologisy. Neuroimaging clin N Am 23(3):381-392

18. Caruso PA, Johnson J, Thibert R, Rapalino O, Rincon S, Ratai E-M (2013) The use of magnetic resonance spectroscopy in the evaluation of epilepsy. Neuroimaging Clin N Am 23(3):407-424

19. Bogdan AA, Kataeva GV, Khomenko JG, Ilves AG, Prakhova LN (2017) Diagnostic value of short and long echo time in $1 \mathrm{H}-\mathrm{MRS}$ for patients with multiple sclerosis. Appl Magn Reson 48(7):707-714

20. Cendes F, Knowlton RC, Novotny E, Min LL, Antel S, Sawrie S et al (2002) Magnetic resonance spectroscopy in epilepsy : clinical issues. Epilepsia 43: 32-39

21. Campos BAG, Yasuda CL, Castellano G, Bilevicius E, Li LM, Cendes F (2010) Proton MRS may predict AED response in patients with TLE. Epilepsia 51(5): 783-788

22. Briellmann RS, Mark Wellard R, Masterton RAJ, Abbott DF, Berkovic SF, Jackson GD (2007) Hippocampal sclerosis: MR prediction of seizure intractability. Epilepsia 48(2):315-323

23. Laxer KD, Trinka E, Hirsch LJ, Cendes F, Lang J, Delanty N et al (2014) The consequences of refractory epilepsy and its treatment. Epilepsy \& Behavior 37:59-70

24. Kasper BS, Struffert T, Doelken MT, Mennecke A, Stadlbauer A, Kecskeme L et al (2010) Multi-voxel magnetic resonance spectroscopy at $3 \mathrm{~T}$ in patients with idiopathic generalized epilepsy. Seizure 19:485-492

\section{Publisher's Note}

Springer Nature remains neutral with regard to jurisdictional claims in published maps and institutional affiliations.

\section{Submit your manuscript to a SpringerOpen ${ }^{\circ}$ journal and benefit from:}

- Convenient online submission

- Rigorous peer review

- Open access: articles freely available online

High visibility within the field

- Retaining the copyright to your article

Submit your next manuscript at $\boldsymbol{\nabla}$ springeropen.com 